\title{
Topology Optimization Design and Thermodynamic Analysis of the Missile Engine Support Structure Based on Numerical Simulation and 3D Printing
}

\author{
Miao-Wen Jiang \\ Laser Engineering Research Institute, \\ Beijing University of Technology, \\ Beijing Digital Medical 3D Printing Engineering \\ Technology Center \\ Beijing, China \\ E-mail: jiangmiaowen415@163.com
}

\author{
Jian-Zhuo Yan \\ Faculty of Information Technology, \\ Beijing University of Technology, \\ Beijing Digital Medical 3D Printing Engineering \\ Technology Center \\ Beijing, China \\ E-mail: yanjianzhuo@bjut.edu.cn
}

\begin{abstract}
This paper aims to offer a lightweight design method of the missile engine support structure based on the topology optimization by taking advantage of the computer numerical simulation and 3D printing technology. Taking use of 3D printing in the shape of heterogeneous structures and personalized manufacturing advantages, a design process is proposed including a computer modeling, numerical simulation, 3D printing and engineering verification of the missile engine lightweight structure. Based on the finite element method, a topology optimization algorithm based on the minimum potential energy of structure is proposed. The design methodology has the following aspects: first, the design object needs to complete three-dimensional modeling by a computer aided design (CAD) software (UG); then, the topology optimization model was established by a computer aided analysis (CAE) software (HyperWorks); finally, the topology optimization results are fed back into the design for the final lightweight design model and $3 D$ printing. In terms of analytical verification, the stress and displacement results of the topology optimization model are examined by numerical simulation; this paper uses HyperWorks for thermodynamic numerical simulation; the maximum bearing capacity of the structure is analyzed by engineering test. The experimental results show that this study can achieve the purpose of lightweight in the case of certain constraints, while weight loss effect can reach $11.06 \%$. In addition, 3D printing technology combined with numerical simulation can shorten the development cycle and improve the design efficiency.
\end{abstract}

Keywords-numerical simulation; $3 D$ printing; topology optimization; thermodynamic analysis; missile engine support structure

\section{INTRODUCTION}

3D printing technology is subordinate to the scope of non-traditional processing technology. The technology includes electromechanical, optical, computer, control and materials and other disciplines. 3D printing technology is an innovation in the world's advanced manufacturing. Different from the reduced material manufacturing, 3D printing technology can make liquid polymers, powder and other sheet, filamentous discrete materials pile up in the way of layer by layer. The final model is formed in a one-shot fabrication. 3D printing technology in the design of the model need to use computer aided design (CAD) software for rapid modeling. This is in line with modern and future manufacturing trends, with the increasing demand for the product personalization [1]. At present 3D printing technology has the growing demand in the level of designing with an increasingly close integration via CAD and computer aided engineering (CAE).

3D printing technology combined with computer technology will inevitably bring the advantages of the lightweight. Specifically, 3D printing mainly adopts four ways in the structural design to achieve lightweight: the hollow sandwich / thin-walled reinforced structure, the lattice structure, the realization of integrated structure, the topology optimization structure [2].

Topology optimization technology is the redistribution of the original parts of the material, which can be able to achieve functional requirements based on the weight reduction. The topology approach aims to achieve an optimal material distribution within a defined design domain through setting the boundary conditions, the pre-tension and the load as constraints [3]. It can use the topology optimization to determine and remove those parts that do not affect the rigidity of the material, and ultimately achieve lightweight. From the current domestic and international research status, researchers are increasingly aware of the advantages of the lightweight from topology optimization technology when UG, HyperWorks and other computer aided softwares have been applied largely into the industrial design[4] [9]. However in the feedback phase after the computer numerical simulation, researchers often need to complete the re-design by CAD softwares. After the re-design, the result parameters will produce more or less errors, which reduce the accuracy of the parts after the topology optimization. However with the continuous development of 3D printing technology, HyperWorks and other CAE softwares can export the topology-optimized files directly into the default STL format for the 3D printer. It can be re-fed back to the design software for the proper repairing, and finally 3D printing. In summary, this process will minimize the loss of the accuracy of simulation results.

This paper focuses on 3D printing is combined with CAD and CAE. After the topology optimization of the heterogeneous structure through simulation analysis to complete the final modeling, these designs are often unable 
to process through the traditional processing, and through 3D printing can be achieved.

\section{THEORETICAL BASIS AND ALGORITHM DERIVATION}

In the missile structure (Figure 1), the engine support structure is an essential component. In the missile launching state, its support structure will withstand a greater thrust from the engine. Due to the relatively large working load and high temperature, there will be buckling, stress damage, excessive deformation and other potential failure modes [10]. In the practical engineering, the design scheme of the structure is based on empirical design and simplified calculation, which is lack of accurate analysis and is not the optimal scheme.

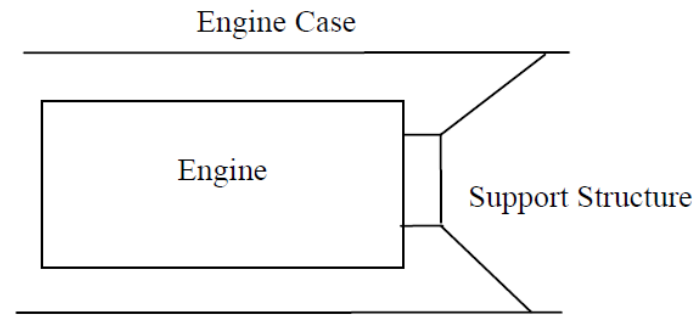

Figure 1. The missile engine support structure.

The support structure of the missile engine is combined with the topology optimization technology. Under the premise of satisfying the rigidity and strength requirements, when the displacement and load are set as the constraint, the topology optimization technology makes the weight of the structure lightest, which saves the material and improves the efficiency of missile launching. In this paper, the OptiStruct module is used to optimize the support structure of the missile engine in the numerical simulation software Hyperworks.

\section{A. Mathematical Model Based on OptiStruct Topology Optimization}

The design variables, objective function and constraints are the three elements of the topology optimization design [11]. In the missile engine support structure studied in this paper, a set of parameters which will be changed in the optimization process to improve the performance are taken as design variables, such as volume and other parameters. The objective function is taken as the optimal design performance index, which is a function of the design variables. Constraints are restrictions on the design variables, such as the displacement and load can be constrained. The mathematical model of the optimal design can be expressed as:

$$
\begin{aligned}
& \text { Minimize: } \\
& f(X)=f\left(x_{1}, x_{2}, \ldots, x_{n}\right) \\
& \text { Subject To: } \\
& g_{i}(X) \leq 0 \quad j=1, \ldots, n \\
& h_{k}(X)=0 \quad k=1, \ldots, m_{h} \\
& x_{i}^{L} \leq x_{i} \leq x_{i}^{U} \quad i=1, \ldots, n
\end{aligned}
$$

$X=x_{1}, x_{2}, \ldots, x_{n}$ represent design variables; $f(X)$ represents the objective function; $g(X)$ represents an inequality constraint function; $h(X)$ represents an equality constraint function; the upper subscript $L$ indicates Lower Limit, the upper subscript $U$ indicates Upper Limit.

After the completion of the mathematical model, OptiStruct mainly uses the local approximation method to solve the optimization problem, the main steps can be divided into:

STEP1: The finite element method (FEM) is used to establish mathematical model;

STEP2:After the iterative calculation, the difference of the target values is smaller than the given convergence tolerance, and the convergence of the problem is solved;

STEP3: To design a sensitivity analysis, which indicates the partial derivative of the design response to the optimization variable;

STEP4: The fitting analysis was performed according to the sensitivity design and the approximate methods can be divided into: the linear simulation, the inverse simulation and the convex simulation [12];

\section{B. FEM based on the Minimum Structural Potential Energy}

According to domestic and foreign literatures on the topology optimization algorithm [13] [16], this paper presents a finite element method based on the principle of the minimization of potential energy, which is based on the topological optimization design of the missile engine support structure. It can be explained that the potential energy of the structure is minimized under the constraint of displacement through the change of the load on the node.

In the FEM analysis, the basic idea is to discretize the continuous structure into a finite number of units, and set a limited number of nodes in each unit. Then, the continuum is regarded as a group of unit aggregates which are continuous only at the node, and the node value of the selected field function is selected as the unknown quantity, and an interpolating function is assumed in the first unit, to represent the distribution of the field functions in the unit. Finally, the field functions on units and even the entire collection are determined by node values and the interpolation function. The analytical method based on the minimum principle of potential energy is deduced as follows:

Firstly, it is necessary to define the field function of the potential energy. According to the principle of the minimization of potential energy in the plane stress, the equation (1) is available:

$$
\delta \prod=0
$$

And the field function $\Pi$ is:

$\Pi=\int_{\Omega} \frac{1}{2}\{\varepsilon\}^{T}[D]\{\varepsilon\} h d \Omega-\int_{\Omega}\{u\}^{T}\{p\} d \Omega-\int_{\Gamma_{\sigma}}\{u\}^{T}\{T\} d \Gamma$ 


$$
\{u\}=\left[\begin{array}{ll}
u & v
\end{array}\right]^{T} \text {------ A force point in the nodal element }
$$
produces both horizontal displacements $u$ and vertical displacements $v$.

$$
\{\varepsilon\}=\left\{\begin{array}{l}
\varepsilon_{x} \\
\varepsilon_{y} \\
\gamma
\end{array}\right\}=\left[\begin{array}{cc}
\frac{\partial}{\partial x} & 0 \\
0 & \frac{\partial}{\partial y} \\
\frac{\partial}{\partial y} & \frac{\partial}{\partial x}
\end{array}\right]\left\{\begin{array}{l}
u \\
v
\end{array}\right\} \text {------ } \varepsilon_{x} / \mathcal{E}_{y} \text { represents the line strain }
$$

in the horizontal and vertical directions, respectively; $\gamma$ indicates a shear strain. Any point in the plane has three strain components and this equation is the relationship between the strain and the displacement.

$$
[D]=\frac{E}{1-u^{2}}\left[\begin{array}{ccc}
1 & u & 0 \\
u & 1 & 0 \\
0 & 0 & \frac{1-u}{2}
\end{array}\right] \text {------ According to (3), the elastic matrix }
$$

$[D]$ in the plane state can be deduced.

$$
\{\sigma\}=[D]\{\varepsilon\}
$$

$$
\{p\}=\left\{\begin{array}{ll}
p_{x} & p_{y}
\end{array}\right\}^{T} \text {------ It represents the unit area load }
$$
in the plane $\Omega$.

$$
\{T\}=\left\{\begin{array}{ll}
t_{x} & t_{y}
\end{array}\right\}^{T} \text {------ It represents the unit length load }
$$
on the plane force boundary $\Gamma_{\sigma}$.

In conclusion, the first term expresses the total strain potential in (1). The latter two terms in (1) show the potential energy of the distributed load, which can be regarded as the external force.

Secondly after the definite field function, the analytical method is to achieve the minimum potential energy of the structure according to the change of the node load under the constraint of the displacement. It is necessary to make the amount of displacement in the boundary not exceed a certain amount of displacement $\{\bar{u}\}$. Displacement $\{u\}$ needs to satisfy (4) in the displacement boundary domain.

$$
\{u\}=\{\bar{u}\}
$$

Third place, the interpolating function can simplify the problem. The displacements within the element can be expressed as an interpolation function (5) for the displacements of the element node. $[N]$ is the matrix of the element shape function

$$
\{u\}=[N]\{\delta\}
$$

According to extremal condition (1) based on the principle of minimum potential energy and combining the mechanical equilibrium equation (6),

$$
[K]\{\delta\}=\{P\}
$$

the field function is derived from the multivariate function for the displacement of the node (7).

$$
\frac{\partial \prod}{\partial \delta_{i}}=0(i=1,2, \ldots, N)
$$
is:

Finally, the expression of the structure potential energy

$$
\Pi=\frac{1}{2}\{\delta\}^{T}[K]\{\delta\}-\{\delta\}^{T}\{P\}
$$

Notes: $\{\delta\}^{T}$------ It means the node displacement at the boundary.

Through the mathematical derivation process, a finite element analysis method based on the minimum potential energy of the structure can be concluded.

\section{A Volume Topological Optimization Algorithm for the Minimum Structure Potential Energy}

In the last section, this paper derives the minimum structural potential energy derivation process, the ultimate goal of which is to achieve the volume optimization under the topology optimization. After the description of this algorithm, in order to ensure that the engine support structure must meet the mechanical properties, the model can achieve the smallest size and achieve the purpose of lightweight. The minimum structural potential topology optimization algorithm for volume is shown:

Input: a finite element mesh model of the missile engine support structure

Output: a volume optimization model for the missile engine support structure

STEP1: The initial volume parameter $V$ and the maximum allowable stress value $\sigma_{\max }$ of the material are determined. According to the equation (2), the minimum structural potential energy under the load and displacement constraints is calculated;

STEP2: To start to optimize the volume, each optimization of the volume of $V_{i}$ needs to calculate the corresponding structural potential energy $\Pi_{i}$ and the maximum stress $\sigma_{i \max }$ of the internal structure;

STEP3: When the maximum stress value $\sigma_{i \max }$ under the structural potential $\prod_{i}$ is less than the maximum allowable stress $\sigma_{\max }$ of the material, the initial volume $V$ can be reduced; 
STEP4: When the maximum stress value $\sigma_{i \max }$ under the structural potential $\prod_{i}$ is equal to $\sigma_{\max }$, the volume is no longer reduced and the optimal volume $V_{\min }$.

STEP5: Return to the STEP1.

\section{Thermodynamic Analysis}

According to the principle of the plane thermal stress, when the object temperature changes, its geometry will be the corresponding changes [17]. A divided grid cell is considered as a plane, and when it is heated and the temperature is changed, each point in the plane has a certain displacement. The strain at this time should be the sum of the elastic strain $\{\varepsilon\}_{E}$ and the thermal strain $\{\varepsilon\}_{T}$, as shown in the equation (9).

$$
\{\varepsilon\}=\{\varepsilon\}_{E}+\{\varepsilon\}_{T}
$$

$\Delta T$ represents the temperature rise and $\alpha$ represents the isotropic linear expansion coefficient, as shown in equation (10).

$$
\{\varepsilon\}_{T}=\left[\begin{array}{lll}
\alpha \Delta T & \alpha \Delta T & 0
\end{array}\right]^{T}
$$

By the equation (3), the relationship between the stress and the total strain can be obtained, as shown in the equation (11).

$$
\{\sigma\}=[D]\left(\{\varepsilon\}-\{\varepsilon\}_{T}\right)
$$

\section{NUMERICAL SIMULATIONS AND TOPOLOGY OPTIMIZATION DESIGN}

\section{A. Topology Representation Based on the Unit Density}

Topological optimization of the material based on OptiStruct will take the unit density as a design variable in the design domain of the finite element model. The unit density has a functional relationship of the elastic modulus $E$ of the material, as shown in Figure 2.

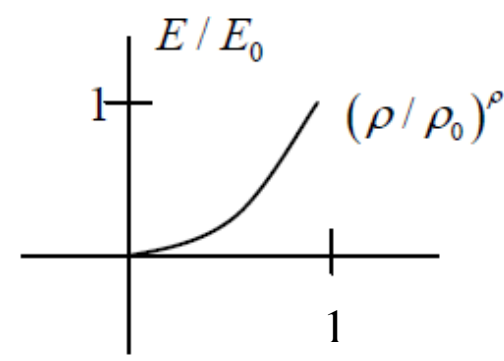

Figure 2. The relationship between the unit density and elastic modulus of the material.
As shown in Fig. 3, the inner red region of the U-shaped fixture is subjected to a force according to the force cloud pattern (a). When the relationship of $\rho-E$ curve in Fig. 2 is given, the result of optimization shows that the material of the red region (the unit density close to 1) forms the transmission path which needs to be retained in the design, while the blue region (the unit density close to 0 ) can be removed.

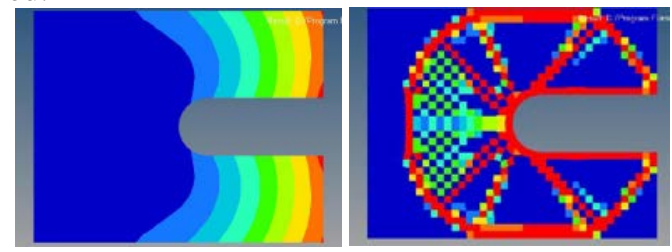

(a) the force cloud pattern (b) Optimization Results

Figure 3. Topology representation.

\section{B. Optimization Design Based on OptiStruct}

In this paper, the prototype of the product is designed by UG, and meshed by HyperMesh. The whole parts are made of the rare earth magnesium alloy and its constitutive relationship is the linear elasticity, and the elastic modulus is $E=45 G P a$, the Poisson's ratio is $\mu=0.35$, the density is $1.8 e+3 \mathrm{~kg} / \mathrm{m}^{3}$. As the topology optimization can not take the stress or strain as a constraint or target, so the whole part of the stiffness can be optimized as the goal and the maximum displacement is less than 1 [18].

Fig. 4 is a finite element model for the topology optimization. The support structure at the bottom of the missile is set by six degrees of freedom constraints and the thrust load acts evenly on the annular front face of the engine. Because the load of the missile engine support structure is the surface load, the surface load is applied to the center of a unit grid. Each load is set to $10 \mathrm{~N}$ and the model is divided into 1253 nodes altogether, so the thrust load of the annular front face is $12530 \mathrm{~N}$.

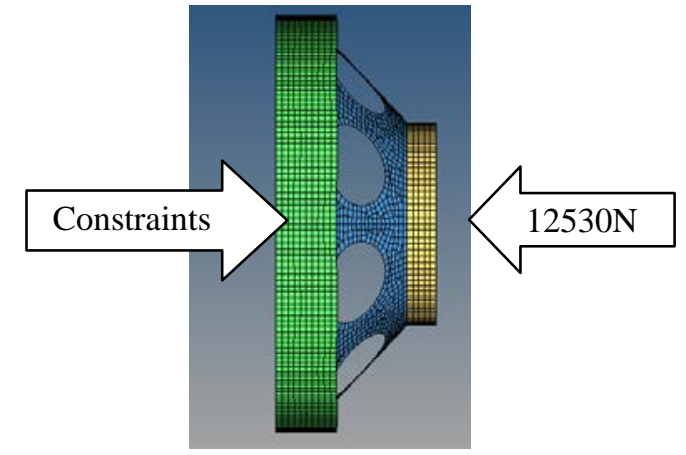

Figure 4. The topological optimization finite element model.

OptiStruct is used to optimize the support structure of the missile engine. The optimization iteration is converged after 6 steps, the constraints are all satisfied and the target is minimized. The overall optimization trend is shown in Figure 5. 


\section{$\triangle$ ATLANTIS PRESS}

According to the topological optimization algorithm with the minimum potential energy, the displacement response and stress variation are taken as references. The results of the last iteration show that the maximum displacement at the annular section is $5.442 \mathrm{e}-1$ and less than the design constraint, and the maximum stress at the optimization is $6.831 \mathrm{~N}$. In the optizamation model, the circular weightreducing holes become long holes with wide in the center and narrow at both ends, as shown in Fig.6 and Fig.7.

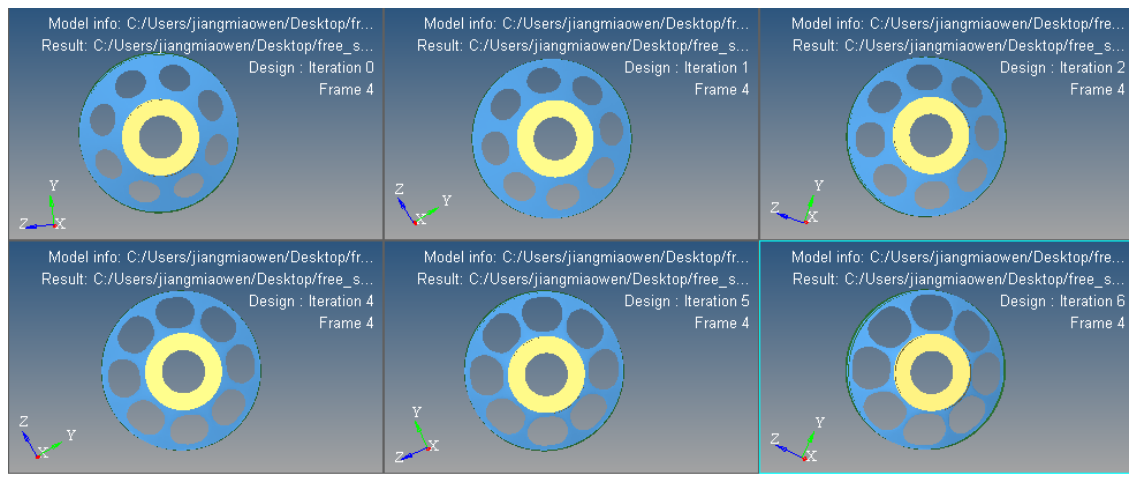

Figure 5. The topological optimization results.

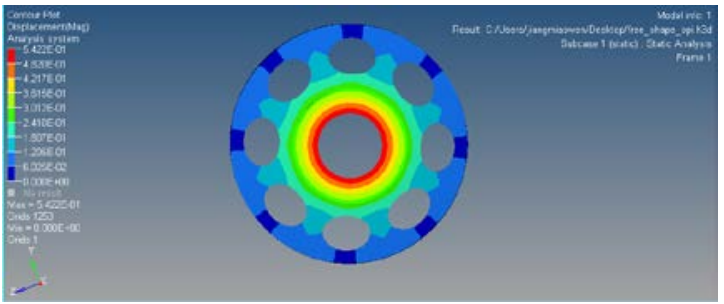

Figure 6. The displacement cloud pattern

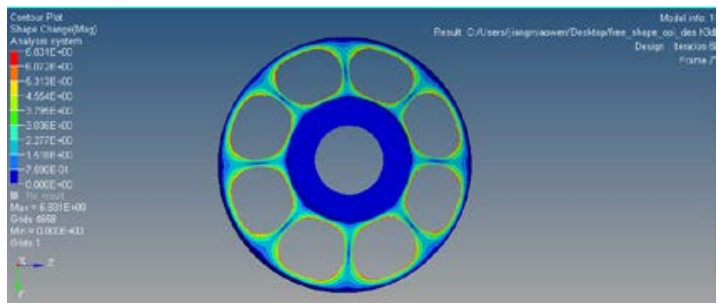

Figure 7. The force cloud pattern

\section{EXPERIMENT AND DISCUSSION}

\section{A. Thermodynamic Analysis Based on RADIOSS}

After the topology optimization, it is necessary to carry out appropriate experiments on the missile engine support structure. The working environment of the missile engine support structure is high temperature and high pressure, so a proper thermodynamic analysis is necessary. The thermodynamic analysis of the finite element model after the topology optimization is carried out by RADIOSS module in HyperWorks. The purpose is to compare the thermodynamic properties of the original model and the optimized model, and to provide some reference values for the feedback design.

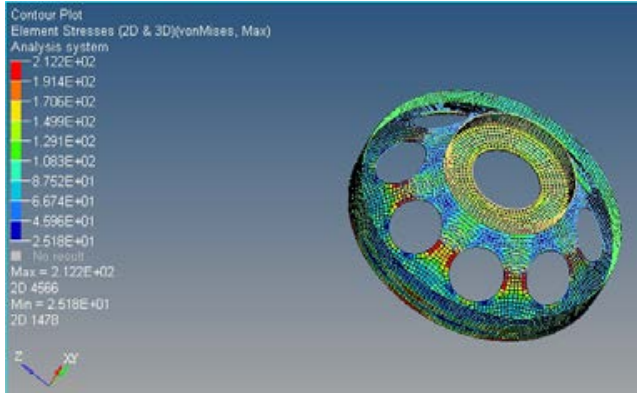

(a) The original model
Therefore, RADIOSS uses the displacement and the stress cloud patterns to carry on the analysis contrast, as shown in Fig.8 and Fig.9.

The force of the connection between the weight-reducing holes increases after the topology optimization, as shown in Fig.8 ; the displacement of the annular end face increases after the topology optimization, as shown in Fig.9.

Therefore, the analysis results can be fed back to the design, the annular end face is designed to be thickened to a certain extent on the basis of the topology optimization. The redesign can be appropriate to reduce the size of holes, so as to increase the area of the connecting portion, thereby forming a final model.

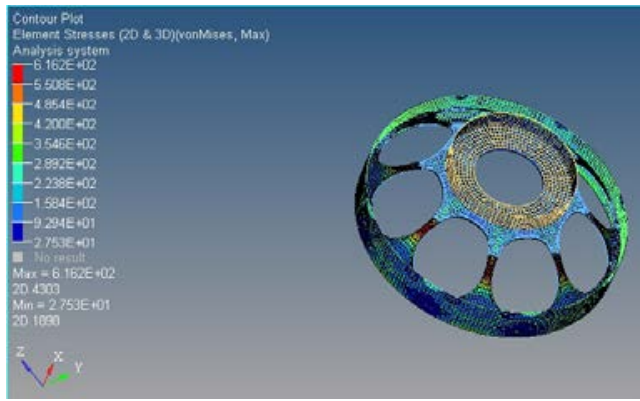

(b) The optimization model

Figure 8. The thermal cloud pattern 


\section{ATLANTIS PRESS}

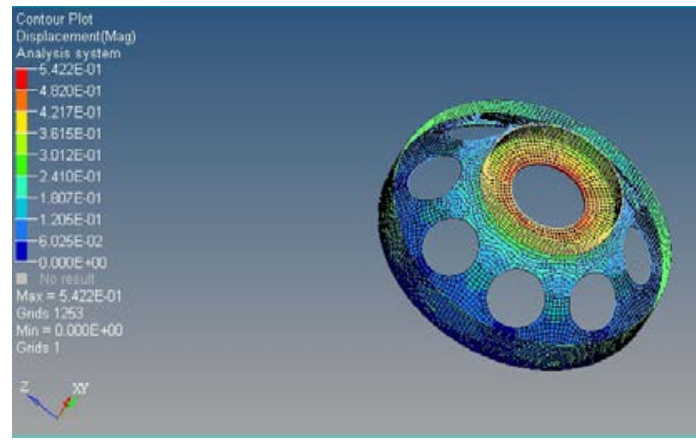

(a) The original model

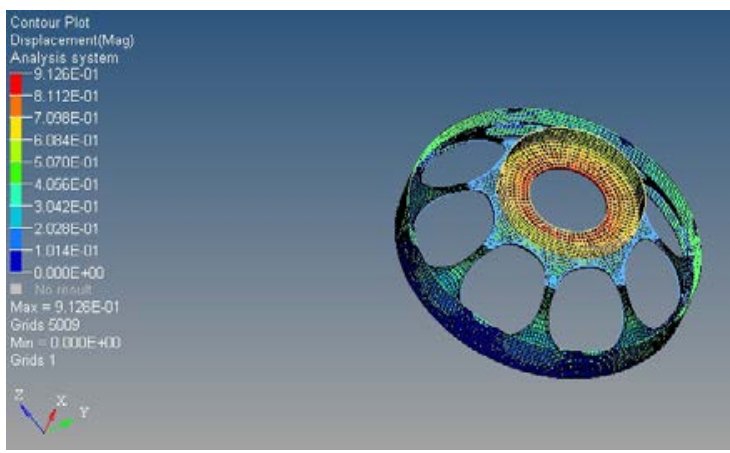

(b) The optimization model

Figure 9. The thermal displacement cloud pattern

\section{B. Manufacturing Process and Engineering Verification}

This experiment uses SPS300 laser light solidification rapid prototyping machine and the printing material is the photosensitive resin. The 3D printing model is shown in Fig.10.

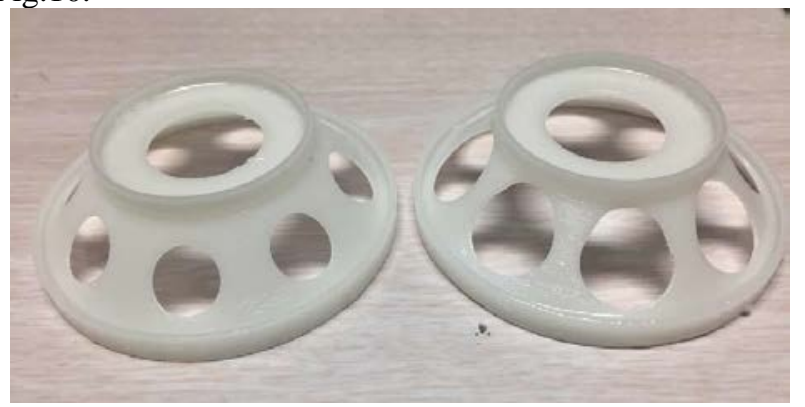

Figure 10. 3D printing model

This engineering verification uses a spiral force test bench and the corresponding data acquisition software to test the 3D printing model for mechanical properties, as shown in Fig.11.

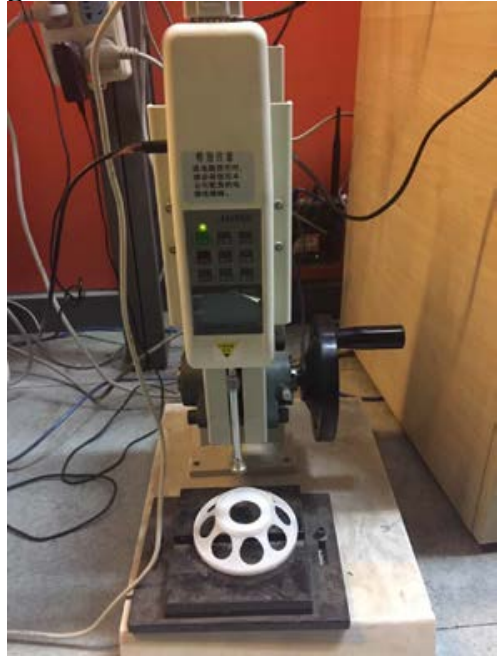

Figure 11. Model stress testing.

The test mainly tests the maximum tolerance for the original model and the optimized model before the failure.
First of all, it is necessary to select the appropriate pressure head to apply the vertical load to the annular end face, then to shake the turntable and gradually compact the model. Through the data acquisition software which is matched with the test bench, the data is extracted and the corresponding data is reduced. Fig.12 shows the relationship between the time and pressure. The maximum pressure that the original structure can bear is in the range of $155 \sim 158 \mathrm{~N}$, and the optimization model is between 142 $\sim 144 \mathrm{~N}$. It can be seen that the variation of the maximum stress of the optimization model is controlled within $10 \%$ of the original model. After the weight comparison, the weight of the original model is $15.5366 \mathrm{~g}$ and the optimized model weight is $13.8182 \mathrm{~g}$, while the weight loss reaches $11.06 \%$ of the original model, as shown in Fig.13.

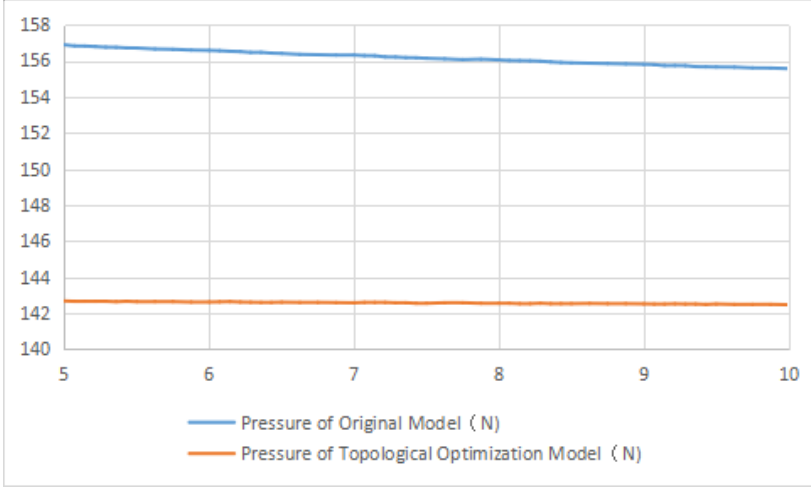

Figure 12. The stress test chart.

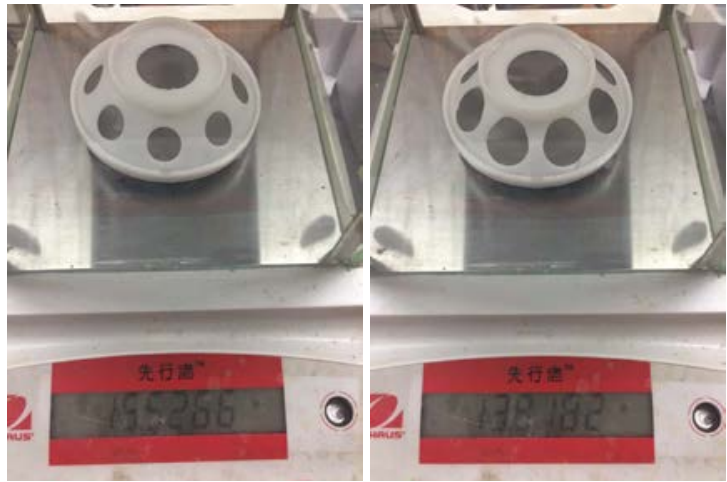

Figure 13. Weight comparison. 


\section{CONCLUSIONS AND FUTURE POSSIBILITIES}

This paper presents a lightweight method for the model of the missile engine support structure using 3D printing.

A complete lightweight design system with the computer modeling, numerical simulation, 3D printing and the engineering verification was built.

Based on the FEM of the minimum potential energy, a topology optimization algorithm based on the minimum potential energy of structure is proposed.

Combined with the corresponding computer software, this paper fully utilizes the advantages of CAD and CAE in the process of $3 \mathrm{D}$ printing.

The experiments show that this research achieves the aim of lightweight design under the condition of satisfying certain constraints. At the same time, 3D printing technology combined with numerical simulation can shorten the development cycle and improve the design efficiency.

The future research will optimize and improve the topology optimization algorithm while improving the accuracy of the topology optimization model into the 3D printing model. In addition, the method is extended to the general model of the lightweight.

\section{REFERENCES}

[1] H. Lipson, M. Kurman, The new world of 3D printing, John Wiley \& Sons, 2013.

[2] L. A. Hockaday, K. H. Kang, N. W. Colangelo, et al. "Rapid 3D printing of anatomically accurate and mechanically heterogeneous aortic valve hydrogel scaffolds," Biofabrication, 2012, 4(3): 035005.

[3] O. Sigmund, J. Petersson, "Numerical instabilities in topology optimization: a survey on procedures dealing with checkerboards, mesh-dependencies and local minima," Structural optimization, 1998, 16(1): 68-75.

[4] K. Suzuki, N. Kikuchi, "A homogenization method for shape and topology optimization," Computer methods in applied mechanics and engineering, 1991, 93(3): 291-318.

[5] X. Huang, S. W. Zhou, Y. M. Xie, “ Topology optimization of microstructures of cellular materials and composites for macrostructures,” Computational Materials Science, 2013, 67: 397-407.
[6] D. J. Munk, G. A. Vio, G. P. Steven, "Topology and shape optimization methods using evolutionary algorithms: a review," Structural and Multidisciplinary Optimization, 2015, 52(3): 613631.

[7] B. Hassani, E. Hinton. Homogenization and structural topology optimization: theory, practice and software. Springer Science \& Business Media, 2012.

[8] R. Rezaie, M. Badrossamay, A. Ghaie, "Topology optimization for fused deposition modeling process,” Procedia CIRP, 2013, 6: 521526.

[9] V. B. Hammer, N. Olhoff, "Topology optimization of continuum structures subjected to pressure loading," Structural and Multidisciplinary Optimization, 2000, 19(2): 85-92.

[10] Z. W. Yang, W. Zhang, G. Tian, "Numerical simulation on thermal wave NDT of missile engine," Nondestructive Testing, 2009, 1: 002.

[11] B. Hassani, E. Hinton, Homogenization and structural topology optimization: theory, practice and software, Springer Science \& Business Media, 2012.

[12] J. M. Melenk, I. Babuška, "The partition of unity finite element method: basic theory and applications," Computer methods in applied mechanics and engineering, 1996, 139(1): 289-314.

[13] P. Hajela, E. Lee, "Genetic algorithms in truss topological optimization,” International journal of solids and structures, 1995, 32(22): 3341-3357.

[14] S. Guindon, O. Gascuel, "A simple, fast, and accurate algorithm to estimate large phylogenies by maximum likelihood," Systematic biology, 2003, 52(5): 696-704.

[15] Q. Bai, "Analysis of particle swarm optimization algorithm," Computer and information science, 2010, 3(1): 180.

[16] S. K. Wang, K. Tai, M. Y. Wang, "An enhanced genetic algorithm for structural topology optimization,” International Journal for Numerical Methods in Engineering, 2006, 65(1): 18-44.

[17] B. Kongtragool, S. Wongwises, "Thermodynamic analysis of a Stirling engine including dead volumes of hot space, cold space and regenerator,” Renewable Energy, 2006, 31(3): 345-359.

[18] C. O. Katsanos, D. T. Hountalas, E. G. Pariotis, "Thermodynamic analysis of a Rankine cycle applied on a diesel truck engine using steam and organic medium,” Energy Conversion and Management, 2012, 60: 68-76. 